Boletín de la Sociedad Geológica Mexicana

VOLUMEN 62, NÚM. 3, 2010, P. 437-451

\title{
Registro de sequías históricas en el occidente de México con base en el análisis elemental de sedimentos lacustres: El caso del lago de Santa María del Oro
}

\author{
Susana Sosa-Nájera ${ }^{1, *}$, Socorro Lozano-García ${ }^{1}$, Priyadarsi D. Roy ${ }^{1}$, Margarita Caballero ${ }^{2}$ \\ ${ }^{1}$ Instituto de Geología, Universidad Nacional Autónoma de México, 04360, México D. F., México. \\ ${ }^{2}$ Instituto de Geofísica, Universidad Nacional Autónoma de México, 04360, México D. F., México. \\ *susosa@servidor.unam.mx
}

\section{Resumen}

Se presentan datos de alta resolución de concentración de titanio (Ti) y calcio (Ca) así como de susceptibilidad magnética de sedimentos lacustres del lago Santa María del Oro, localizado en la porción occidental del centro de México. Las mediciones elementales se realizaron con un escáner FRX ITRAX. El registro de Ti, que abarca los últimos 700 años (1300 a 2000 d. C.), fue validado como indicador de escurrimiento (y por lo tanto de precipitación) con registros históricos y datos de anillos de árboles relacionados con sequías importantes en diferentes regiones de México. Se identifican en el registro de Ti 21 eventos de sequía, destacando seis por su intensidad y/o duración (1365-1384, 1526, 1655-1670, 1818, 1900 y 1930-2000). El resultado del análisis espectral de los datos de concentración de Ti indica la existencia de sequías recurrentes en periodicidades de 25, 39, 50, 70 y 117 años. Dichas periodicidades pueden estar asociadas a la actividad solar y/o a factores climáticos como El Niño. Los eventos de sequía del lago Santa María del Oro se compararon con el indicador del desplazamiento latitudinal de la posición promedio de la Zona de Convergencia Intertropical (el registro de Ti de la cuenca del Cariaco en el Océano Atlántico) y con el índice de El Niño. Algunos de los eventos mayores de sequía identificados (1448-1435, 1485-1474, 1605-1601, 1673-1655 y 1770-1773) se correlacionan con el desplazamiento de la Zona de Convergencia Intertropical (ZCIT) hacia el sur durante el verano, mientras que otros años secos (1818, 1832, 1866, 1900, 1939-1929 y 1958-2000) se correlacionan con un índice positivo de El Niño. Además, se registraron otros eventos de sequía donde se combinan el índice positivo del El Niño con la posición meridional de la ZCIT (1386-1354, 1536-1510, 1579-1585 y 1780-1808).

Palabras clave: sequías, titanio, Holoceno tardío, ITRAX, Zona Intertropical de Convergencia, Monzón Mexicano.

\begin{abstract}
This paper presents high-resolution data of titanium (Ti) and calcium (Ca) concentrations and magnetic susceptibility of lacustrine sediments from Lake Santa Maria de Oro, located in western central Mexico. Major element measurements were carried out with an ITRAXXRF core scanner. The Ti record for the last 700 years (1300-2000 AD) was validated as a marker for wash and runoff events (and therefore precipitation) by comparison with historical information and tree ring chronologies related to major droughts events from several areas in Mexico. The register of Ti identifies 21 drought events, six of which are of major intensity and/or duration (i.e. 1365-1384, 1526, 1655-1670, 1818, 1900 and 1930-2000 AD). Spectral analysis of Ti suggests periodicities of 25, 39, 50, 70 and 117 years for the drought events. These periodicities could be related to solar activity and/or climatic factors such as El Niño Southern Oscillation (ENSO). The drought events documented at Lake Santa Maria del Oro were compared with records of latitudinal displacement of the Intertropical Convergence Zone (the Ti record from Cariaco Basin in the Atlantic Ocean) and the ENSO index. Some of the main drought events (1448-1435, 1485-1474, 1605-1601, 1673-1655 and 1770-1773 AD) correlate with summer southern migration of the Intertropical Convergence Zone (ITCZ), while others, i.e.1818, 1832, 1866, 1900, 1939-1929 and 1958-2000 AD, show a correlation
\end{abstract}


with the ENSO index. The events of 1386-1354, 1536-1510, 1579-1585 and 1780-1808 AD correlate positively both with the ENSO index and southern ITCZ migration.

Keywords: droughts, titanium, late Holocene, ITRAX, ITCZ, Mexican Monsoon.

\section{Introducción}

Durante las últimas décadas, la investigación relacionada con la historia del cambio climático ha sido significativa; en particular, los estudios enfocados a documentar la variabilidad climática del último milenio en diversas regiones del planeta han aportado datos sobre el funcionamiento del sistema climático. Sin embargo, en la síntesis de datos paleoclimáticos presentados por el Panel Intergubernamental de Cambio Climático (IPCC, 2007) es notable la escasez de registros para las regiones tropicales. En el caso de México, aunque hay estudios para la zona central (Ortega-Guerrero et al., 2000; Israde-Alcántara et al., 2002; Caballero et al., 2006, Figueroa-Rangel et al., 2006; Lozano-García et al., 2007; Roy et al., 2008; Vázquez-Castro et al., 2008; Kienel et al., 2009) en la península de Yucatán (Curtis et al., 1996; Hodell et al., 2001, 2005, Haug et al., 2003) y en algunos sitios del norte (Metcalfe et al., 1997; Lozano-García et al., 2002; Barron et al., 2003, 2004; Pérez-Cruz, 2006; Ortega-Rosas et al., 2008) es evidente la falta de registros de alta resolución que permitan establecer con precisión la variabilidad climática de las últimas centurias.

Una fuente de información sobre la historia del cambio ambiental son los sedimentos que se depositan en el fondo de los lagos. En éstos quedan archivadas señales geoquímicas (isótopos, propiedades magnéticas, variaciones elementales) y restos de organismos (polen, ostrácodos, diatomeas) que pueden interpretarse y calibrarse para inferir los cambios climáticos en periodos previos al registro instrumental. Las evidencias sobre cambios hidrológicos en algunas cuencas del centro y norte de México, se han reconstruido con base en los cambios de composición de los conjuntos de diatomeas que se acumulan en los sedimentos. En estos estudios, la dominancia de diatomeas del tipo aerófilo indica la disminución del nivel lacustre y es interpretada como indicador de ambientes más secos (Metcalfe, 1995; Caballero y Ortega-Guerrero, 1998; Caballero et al., 1999; Bradbury, 2000; Israde-Alcántara et al., 2002). Sin embargo, la resolución de estas reconstrucciones paleolimnológicas es generalmente de escala de centurias, por lo que las señales de variación climática decadales o sub-decadales no se detectan.

Un método novedoso en el estudio de secuencias lacustres es la obtención del registro de alta resolución de las variaciones elementales en sedimentos utilizando el escáner de fluorescencia de rayos X (ITRAX) el cual permite inferir variaciones ambientales (Croudace et al., 2006; Rothwell y
Rack, 2006; Rothwell et al., 2006). En particular, el titanio es considerado como un indicador del registro clástico, ya que se encuentra en minerales que no se meteorizan con facilidad. Este elemento es considerado entre los más inmóviles durante los procesos de intemperismo, por lo que su registro sólo responde a aporte clástico. Por otra parte, el calcio pude indicar momentos de menor sedimentación clástica y, por ende, de mayor sedimentación autigénica en el lago, dado que en la cuenca del lago no hay rocas carbonatadas. La precipitación de $\mathrm{CaCO}_{3}$ puede ser inducida por factores bióticos y abióticos, como el incremento en la temperatura, el metabolismo bacteriano y el estado trófico del lago. Así, el calentamiento de las aguas superficiales durante la primavera permite la super-saturación de $\mathrm{CaCO}_{3}$, la cual incrementa la productividad del lago permitiendo el consumo biológico de $\mathrm{CO}_{2}$, lo cual resulta en un aumento del $\mathrm{pH}$. Por otro lado, durante el verano la precipitación de carbonato de calcio produce la acumulación de láminas de carbonato de milímetros de grosor (Kelts y Hsü, 1978; Eugster, 1980; Boyle, 2001). Valores altos de Ti y bajos de $\mathrm{Ca}$ indican un incremento en el aporte de sedimentos terrígenos de la cuenca hacia el lago debido a alta precipitación y descarga por procesos pluviales (Kelts y Hsü, 1978; Eugster, 1980; Boyle, 2001; Haug et al., 2001a; Barron et al., 2003, 2004; Peterson y Haug, 2006; Rothwell y Rack, 2006).

Algunos de los lagos cráter que se localizan en la Faja Volcánica Trans-Mexicana (FVTM) presentan secuencias sedimentarias que son susceptibles de ser analizadas para establecer las tendencias de cambio ambiental estudiando el registro geoquímico. En general, estos lagos tienen una morfología característica con paredes elevadas casi verticales que protegen al cuerpo de agua del viento. Son lagos profundos de fondo plano y de estratificación estacional (monomixis) en los cuales las aguas profundas (hipolimnion) son anóxicas. Debido a esto no hay perturbación de los sedimentos, lo que permite la preservación de estructuras como la estratificación o laminación de los sedimentos (O'Sullivan, 1983). Estas secuencias de sedimentos laminados permiten archivar la señal ambiental en escalas temporales de alta resolución, ya sea anuales o estacionales.

En este trabajo se presentan evidencias de sequías de los últimos 700 años en la zona occidental del centro de México, con base en el análisis elemental, utilizando los datos de alta resolución de titanio y de calcio de sedimentos del lagocráter Santa María del Oro (LSMO). La comparación de las concentraciones de Ti permite establecer correlaciones 
con la información sobre las sequías reveladas en anillos de crecimiento de árboles y documentadas en registros históricos.

\section{2. Área de estudio}

El lago Santa María del Oro (LSMO) se localiza en la parte nor-occidental de la FVTM, en el límite con la porción sur de la provincia fisiográfica Sierra Madre Occidental, al sureste del estado de Nayarit, a $21^{\circ} 22^{\prime} \mathrm{N}$ y $104^{\circ} 34^{\prime} \mathrm{W}$, con una altitud de $750 \mathrm{msnm}$ (Figuras 1A, 1B, 1C). El cráter parece ser una forma geológica reciente, ya que no se reconocen rasgos que indiquen una estructura pre-existente (Nelson y Sánchez-Rubio, 1986; Vázquez-
Castro, 2004). Al presentarse alineado con conos asociados al volcán Sangangüey se puede inferir que tiene una edad pleistocénica (Ferrari et al., 1997).

El LSMO tiene un diámetro aproximado de $2 \mathrm{~km}$ y un área de $3.7 \mathrm{~km}^{2}$ con una profundidad máxima de $65.5 \mathrm{~m}$ (Serrano, 2004; Figura 1C). Es un lago cráter, endorreico, alimentado por arroyos de escurrimiento intermitente, los cuales están activos únicamente durante la época de lluvias. La erosión del interior del cráter ha propiciado un drenaje de tipo dendrítico y radial convergente hacia el lago (VázquezCastro, 2004). La elevación que rodea al lago es de 300 a $400 \mathrm{~m}$ en las riberas norte y sur respectivamente.

El lago permanece estratificado la mayor parte del año (julio a noviembre) y presenta el mayor gradiente térmico entre los 15 y $20 \mathrm{~m}$ de profundidad. El lago se mezcla de

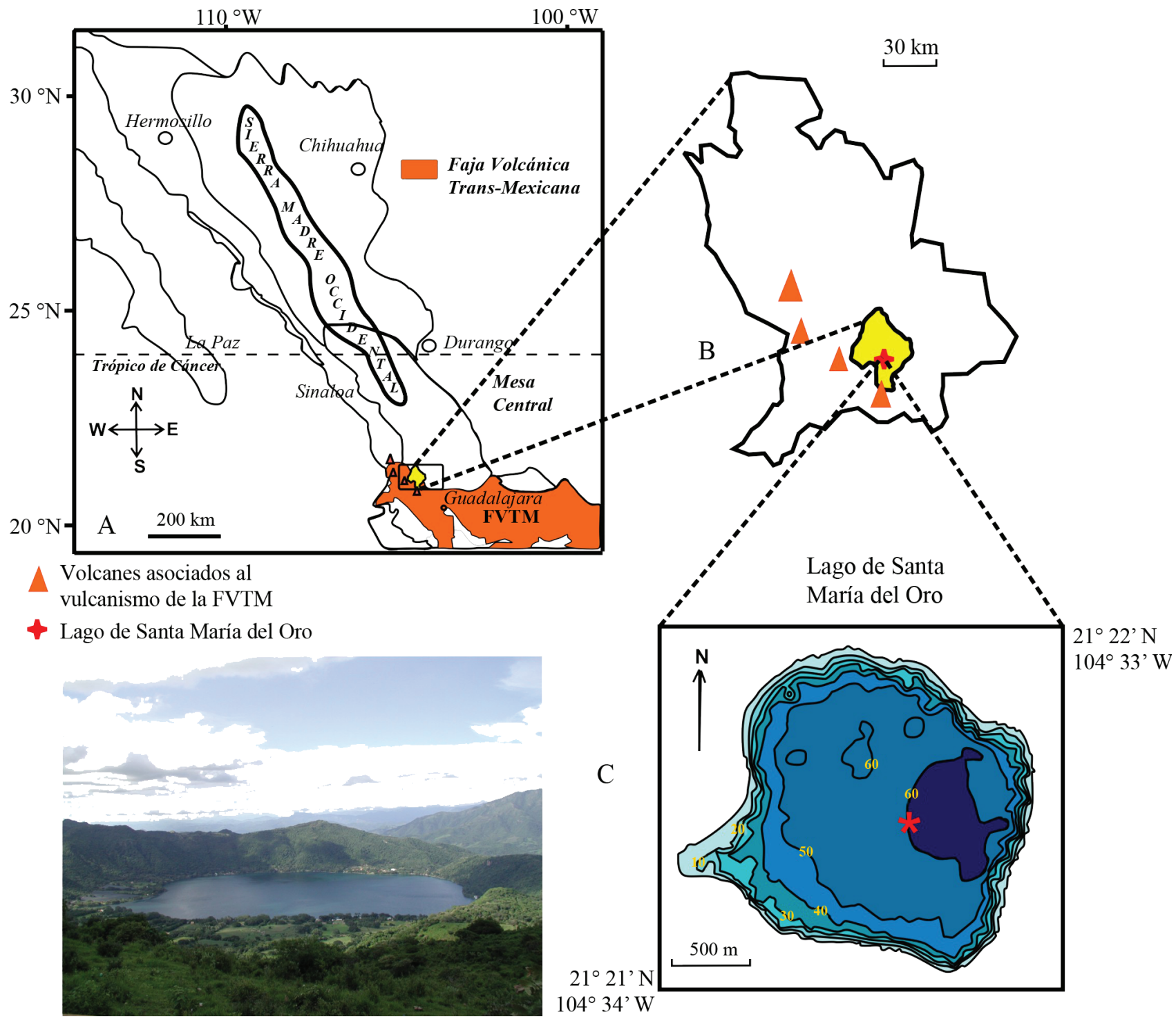

Figura 1. A) Mapa de localización del área de estudio: Sierra Madre Occidental en su intersección con la Faja Volcánica Trans-Mexicana (modificado de Ferrari, 2007). B) Estado de Nayarit con el municipio de Santa María del Oro (amarillo) y localización del lago. C) Mapa batimétrico y fotografía del lago Santa María del Oro. En amarillo se indican las profundidades batimétricas (en metros); el asterisco rojo indica el sitio de perforación. 
febrero a marzo cuando tiene un perfil de temperatura casi homogéneo, por lo cual se clasifica como un lago monomíctico cálido (Serrano, 2004; Filonov et al., 2005). La productividad biológica es baja excepto en los meses más cálidos (abril-agosto), cuando se producen florecimientos algales. El pH del agua del lago es de 7 a 9, el Eh de 0 a $350 \mathrm{mV}$ y presenta una conductividad eléctrica $(\Omega)$ es de $1450 \mu \mathrm{S} / \mathrm{cm}$. Los iones dominantes son los carbonatos, los bicarbonatos y los cloruros, en el campo de los aniones, y el sodio y el magnesio dominan en el campo de los cationes (Armienta et al., 2008).

El clima de la región está controlado por el desplazamiento anual de la ZITC. La posición, intensidad y densidad de la convección en el Pacífico tropical puede resultar en periodos de fuertes lluvias o severas sequías para México (Magaña et al., 2003; Méndez y Magaña, 2010). Durante el verano boreal, la ZITC se desplaza hacia el norte, aproximadamente $10^{\circ}$, en respuesta al incremento de insolación en el hemisferio norte. A principios de primavera comienza a incrementase la temperatura superficial del océano (TSO) en más de $26^{\circ} \mathrm{C}$, y, con el desplazamiento de la ZITC a una posición más boreal, se dispara el establecimiento del Monzón Mexicano (Wang y Fiedler, 2006), el cual se caracteriza por fuertes lluvias durante el verano. Éstas se dispersan hacia el Norte sobre las laderas de la Sierra Madre Occidental a principios de julio, la precipitación por convección se incrementa coincidiendo con el incremento en la humedad y los vientos del sur fluyen hacia el Golfo de California (Higgins et al., 1997).

Específicamente en el LSMO el clima es tropical subhúmedo $\left(\mathrm{Aw}_{\mathrm{o}}\right)$, que se caracteriza por presentar lluvias en verano (junio a octubre) y una temporada de secas que se extiende durante 6 a 8 meses. La precipitación media anual es de $1214 \mathrm{~mm}$ y la temperatura media anual es $21^{\circ} \mathrm{C}$, el mes más cálido es mayo, con una temperatura máxima mensual de $36^{\circ} \mathrm{C}$ y el más frío es enero, con una temperatura mínima mensual de $3.5^{\circ} \mathrm{C}$. Las lluvias en el LSMO están relacionadas con la entrada de vientos del este (alisios) que traen humedad del Golfo de México y con el sistema del Monzón Mexicano (Adams y Comrie, 1997) que aporta humedad del Océano Pacífico tropical. Aproximadamente del $70 \%$ al $80 \%$ de la lluvia anual que se produce en los meses de julio a septiembre en esta región es aportada por el Monzón Mexicano (Serrano, 2004). El evento de El Niño está relacionado con una menor actividad convectiva en el Pacífico mexicano así como una menor cantidad de lluvias, produciendo con frecuencia sequías, con efectos asociados como menor humedad en el suelo y la consecuente pérdida de bosques y cosechas (Magaña et al., 1999). Estudios recientes indican que en años en que ocurre El Niño, el Monzón tiende a retrasarse o a adelantarse con respecto a la fecha de inicio de la temporada de lluvias, lo que significa una temporada de lluvias deficiente y/o el debilitamiento del Monzón en el verano (Higgins et al., 1997; O'Hara y Metcalfe, 1997).

\section{Metodología}

En la parte central del LSMO (60 $\mathrm{m}$ de profundidad) fueron perforados cinco núcleos con una longitud aproximada de $9 \mathrm{~m}$ cada uno. Para ello se utilizó un nucleador de gravedad tipo Kullemberg del Limnological Research Center (LRC) de la Universidad de Minnesota. Cada núcleo fue seccionado en tramos de $1 \mathrm{~m}$ para facilitar su manejo y traslado. Los tramos fueron documentados en el LRC, esto incluyó el uso de un multisensor con un Geotek Core Logger (MSCL) con el que se midió la densidad por atenuación de rayos gamma, la susceptibilidad magnética de alta resolución, la velocidad y amplitud de ondas $\mathrm{P}$ y la porosidad. Posteriormente, los núcleos fueron fotografiados usando una cámara tipo line-scan unida al transportador de núcleos del MSCL (sensor Geoscan IV). Con base en estos datos, los tramos fueron correlacionados para formar una secuencia maestra que en su totalidad presenta una longitud de $9 \mathrm{~m}$.

En los núcleos que integran la secuencia maestra se seleccionaron las muestras para fechamiento por radiocarbono. Así, el marco cronológico de la secuencia se basa en cinco fechamientos con radiocarbono por espectrometría de masas con acelerador (AMS, accelerator mass spectrometer) realizados en muestras de sedimento total. Las fechas obtenidas (Tabla 1) fueron calibradas a años calendáricos con Calib 5.0 y con la base de datos IntCal 04 (Stuiver y Reimer, 1993; Reimer et al., 2004; Stuiver et al., 2005).

Las concentraciones elementales fueron medidas en todos los núcleos que constituyeron la secuencia maestra. Para esto se utilizó el equipo ITRAX de fluorescencia de Rayos X, éste es un método no destructivo el cual permite analizar las variaciones elementales en secuencias sedimentarias continuas obteniendo información geoquímica de alta resolución. El análisis elemental se realizó con el ITRAX de la Universidad de Southampton, Inglaterra, a lo largo de los núcleos de la secuencia maestra con un intervalo de $1 \mathrm{~mm}$. Para este estudio se presentan únicamente las variaciones de $\mathrm{Ti}$ y $\mathrm{Ca}$ de la parte superior de la secuencia maestra que corresponde a los primeros 160 $\mathrm{cm}$. Dado que los datos del ITRAX son semi-cuantitativos y producidos como intensidades, conteos por segundo (cps; Croudace et al., 2006), éstos se transformaron a valores de concentración en porcentaje. Para ello fueron seleccionadas 16 muestras en diferentes niveles de la sección maestra y se analizaron por el método de fluorescencia de rayos $\mathrm{X}$ (FRX) tradicional. El criterio empelado para la selección de muestras fue elegir niveles que presentaran diferentes intensidades de Ti. La concentración elemental fue analizada en el Laboratorio Universitario de Geoquímica Isotópica (LUGIS) del Instituto de Geología (UNAM) con un equipo de FRX Siemens SRS 3000, siguiendo el método de Verma et al. (1996). Los valores de intensidad (cps) de Ca y Ti que se obtuvieron con el ITRAX, fueron transformados 
a concentración utilizando los datos del análisis de FRX tradicional realizados en las muestras seleccionadas. Así, a través de una regresión lineal entre los datos de intensidad contra concentración (\%, FRX) los datos de intensidad de Ti y Ca se calibraron a concentración (Figura 2). Esta transformación de los valores de intensidad del ITRAX, se hizo utilizando la relación:

donde:

$$
\mathrm{y}=\mathrm{mx}+\mathrm{c}
$$

$\mathrm{y}=$ concentración analizada por FRX,

$\mathrm{x}=$ intensidad de cada elemento medido en el ITRAX,

$\mathrm{m}=$ pendiente de la recta de cada elemento $\mathrm{y}$

$\mathrm{c}=$ ordenada al origen.

Con el fin de determinar la existencia de ciclicidad en los datos de concentración de Ti se llevo a cabo un análisis espectral. Esto fue realizado asignando edades a los datos de concentración de Ti. La serie de tiempo fue analizada utilizando con MATLAB 7 (MathWorks, 2004) con el propósito de determinar las periodicidades en el registro de Ti así como para identificar la ocurrencia de eventos de mayor y menor humedad en el área y compararla con
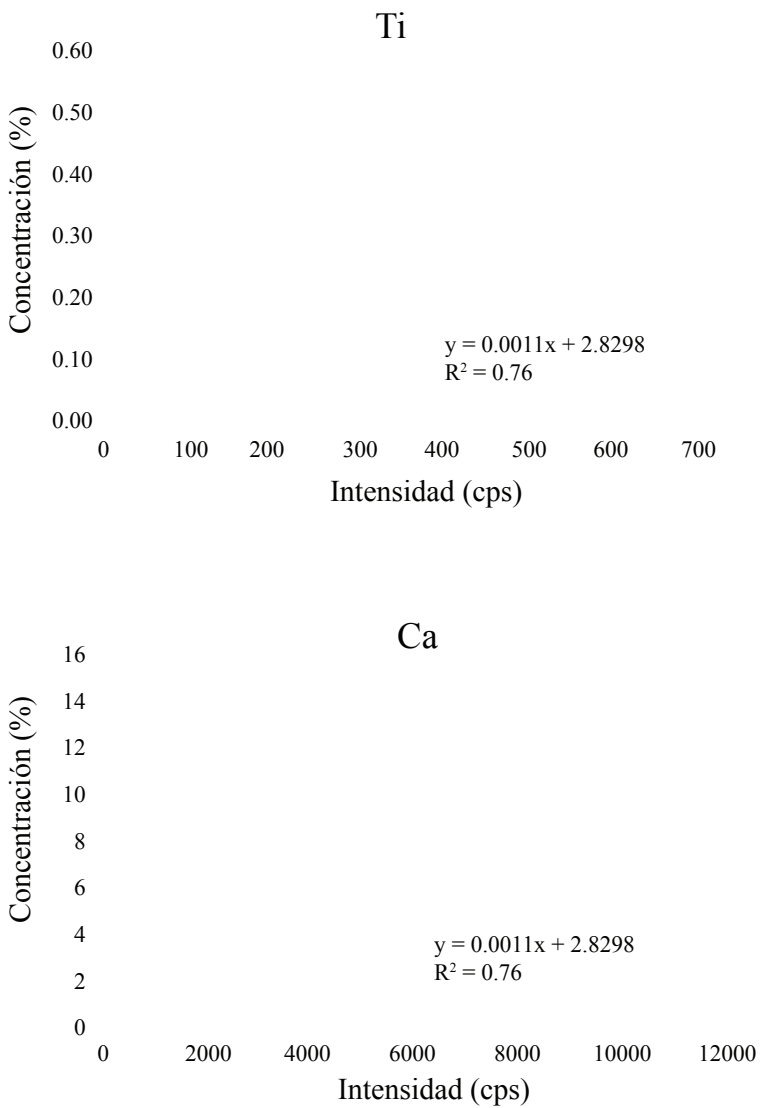

Figura 2. Modelos de regresión lineal entre los valores de ITRAX (cps) y XRF (\%) para Ti y Ca, mostrando en ambos casos altos coeficientes de correlación. Con estos datos se realizó la transformación a concentración $(\%)$. otros estudios.

\section{Resultados}

\subsection{Cronología}

Los resultados de la cronología que se estableció con base en cinco fechas de radiocarbono (AMS) en sedimento total, se presentan en la Tabla 1. La edad de la superficie de los sedimentos se asumió en 2003 cuando el núcleo fue colectado, extrapolándose una edad para la base de la secuencia de 4040 años cal aP (antes del presente) que corresponde al año 2573 a. C. (antes de Cristo), lo que indica que la secuencia abarca todo el Holoceno tardío (Figura 3). Con base en este modelo de edad, los datos de los primeros $160 \mathrm{~cm}$ que aquí se presentan abarcan los últimos 700 años, de 1300 a 2000 d. C. y a partir de aquí se indican en el texto en años de calendario (después de Cristo).

\subsection{Estratigrafía}

La parte superior (primeros $160 \mathrm{~cm}$ ) de la secuencia sedimentaria compuesta de $9 \mathrm{~m}$ esta laminada, cuya estratigrafía se resume a continuación.

De $0 \mathrm{~cm}$ a $160 \mathrm{~cm}$, los sedimentos están bandeados, alternando de negro a gris obscuro (oxidando a café rojizo obscuro); hay limos arcillosos y oozes de diatomeas. Las láminas obscuras presentan materia orgánica amorfa y algunas testas de Phacotus. Hay capas gruesas de $(2-5 \mathrm{~cm})$ y capas delgadas (1-2 mm) de limo café rojizo obscuro, limos sideríticos y calcáreos y arcillas; estas últimas en capas delgadas intensamente deformadas.

\subsection{Análisis elemental}

Para establecer la relación entre Ti y Ca se representó en forma gráfica su concentración (Figura 4). Como se observa, estos elementos presentan una alta correlación inversa $(r=-0.9)$. Las variaciones en los datos de concentración

Tabla 1. Edades de radiocarbono por espectrometría de masas con acelerador (AMS) en sedimentos orgánicos en cinco muestras del LSMO.

\begin{tabular}{lcll}
\hline $\begin{array}{l}\text { Clave } \\
\text { Lab. Beta }\end{array}$ & Prof. (cm) $\begin{array}{c}\text { Edad } \\
\text { calibrada } \\
\text { años }{ }^{14} \mathrm{C} \text { aP }\end{array}$ & Calibración de $2 \sigma$ \\
\hline 198312 & 170 & $750 \pm 40 \mathrm{aP}$ & Cal d. C. 1220-1300 \\
227823 & 180 & $780 \pm 40 \mathrm{aP}$ & Cal d. C. $1200-1280$ \\
198313 & 368 & $2010 \pm 40 \mathrm{aP}$ & Cal a. C. $160-90$ \\
227825 & 510 & $970 \pm 40 \mathrm{aP}$ & Cal a. C. $1050-890$ \\
198315 & 733 & $2235 \pm 40 \mathrm{aP}$ & Cal a. C. $2470-2200$ \\
\hline
\end{tabular}

Calibración basada en Stuiver y Reimer (1993) y Stuiver et al. (2005). aP $=$ antes del presente; a. $\mathrm{C} .=$ antes de Cristo. 


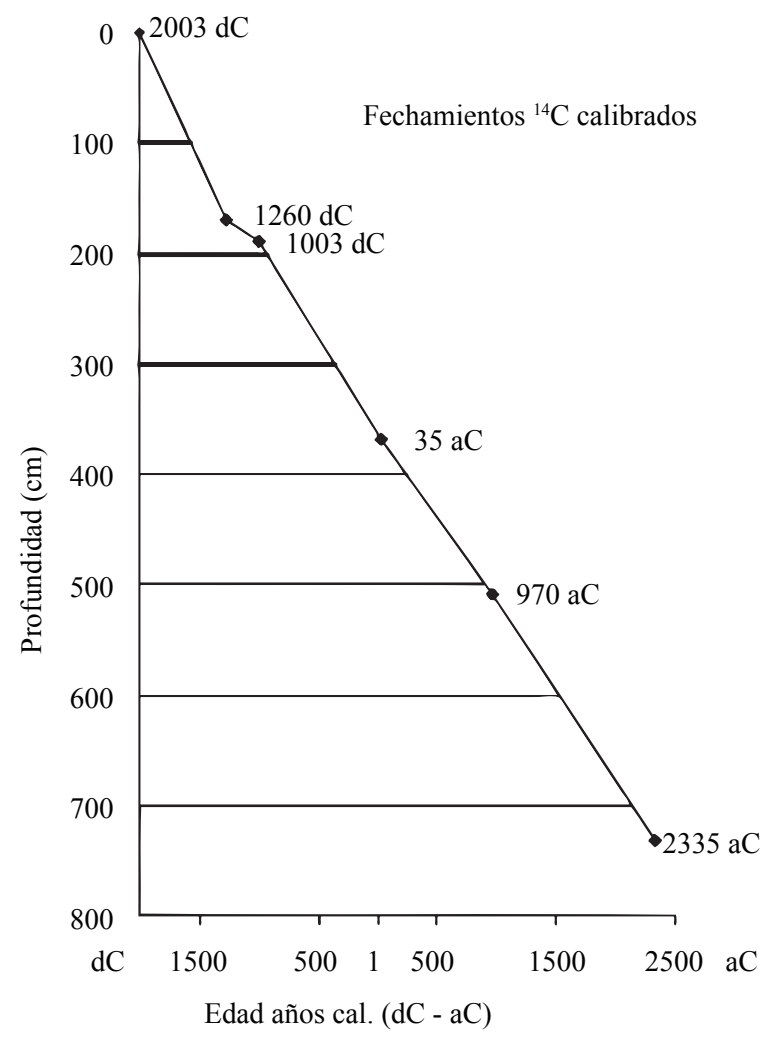

Figura 3. Modelo de edad para la secuencia maestra MOLE SMO03 en edades calibradas a. C./d. C., construidas con los valores medios del intervalo de probabilidad $2 \sigma$.

de Ti y Ca y de susceptibilidad magnética de los últimos 700 años $(160 \mathrm{~cm})$ del LSMO se presentan en la Figura 5. En la secuencia del LSMO se observan variaciones en la concentración de Ti que van de $0.18 \%$ a $0.65 \%$. En general, los valores bajos de Ti en general ocurren cuando hay valores bajos de susceptibilidad magnética y concentración alta de $\mathrm{Ca}$. Estos eventos de disminución en la concentración de Ti sugieren la existencia de periodos de menor aporte clástico al lago y por ende periodos de menor precipitación. Los valores altos indican condiciones de mayor humedad y se asocian con valores altos de susceptibilidad magnética. Con base en la cronología de la secuencia se asignaron las edades a los eventos de baja concentración de Ti como se muestra en la Figura 5, donde destacan 6 periodos de baja concentración de Ti durante el periodo de 700 años (13002000). Estas etapas de menor humedad varían en intensidad y pueden abarcar uno o varios años: 1365-1384, 1526, 1655 $1670,1818,1900$ y 1930-2000. Además, se detectan entre estos periodos mayores de baja concentración de Ti eventos menores que indican menor humedad y que coinciden con periodos históricos y/o de anillos de árboles reportados como épocas de baja precipitación (Figura 6).

El resultado del análisis espectral de los datos de alta resolución de concentración de Ti que se muestran en la Figura 7 indica la existencia de periodicidades de 25, 39, 50,70 y 117 años en el intervalo analizado (1300 a 2000).

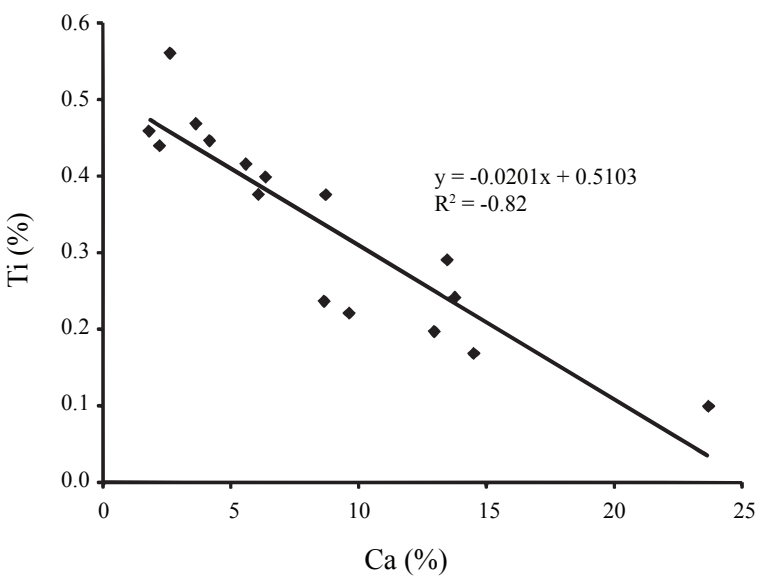

Figura 4. Modelo de regresión lineal entre las concentraciones de Ti (\%) y $\mathrm{Ca}(\%)$, donde se observa una relación inversa con un alto coeficiente de correlación.

Estas periodicidades, que sugieren eventos cíclicos de menor aporte en el Ti, se pueden interpretar como sequías recurrentes. Los mecanismos que determinan estas dichas ciclicidades pueden estar relacionados con actividad solar, y/o fenómenos climáticos.

\section{Discusión}

En el caso de los lagos de cuencas cerradas, las concentraciones de solutos y los niveles lacustres fluctúan en respuesta a la relación precipitación/evaporación $(\mathrm{P} / \mathrm{E})$. Estos cambios en el balance hidrológico son registrados en las facies sedimentarias y en la composición multi-elemental (Valero-Garcés et al., 1997). El Ti es uno de los elementos más inmóviles durante el proceso de intemperismo, por lo que ha sido utilizado como un indicador de cambios en el aporte clástico y puede ser indicativo de cambios en las condiciones hidrológicas (Peterson et al., 2000; Haug et al., 2001a, 2003; Barron et al., 2003, 2004; Croudace et al., 2006; Peterson y Haug, 2006; Rothwell y Rack, 2006; Rothwell et al., 2006). En el registro de Ti del LSMO (Figura 5) se detectan varios eventos con valores bajos en su concentración, los cuales son indicativos de disminución del aporte clástico a la cuenca y decremento en la precipitación.

Con el propósito de comprobar la existencia de eventos de sequías para épocas anteriores a las instrumentales con base en el registro de Ti y compararlo con otras zonas de México, fue necesario buscar fuentes de información paleoambiental alternas, dado que no hay datos de alta resolución disponibles provenientes de algún otro indicador en sedimentos lacustres. Es así que la ocurrencia de episodios secos interpretados a partir de los valores calibrados de Ti puede ser validada con base en documentos históricos (cuando estén disponibles) así como en datos de anillos de crecimiento de árboles. Para este fin, se llevó a cabo una recopilación de este tipo de datos que documentan 


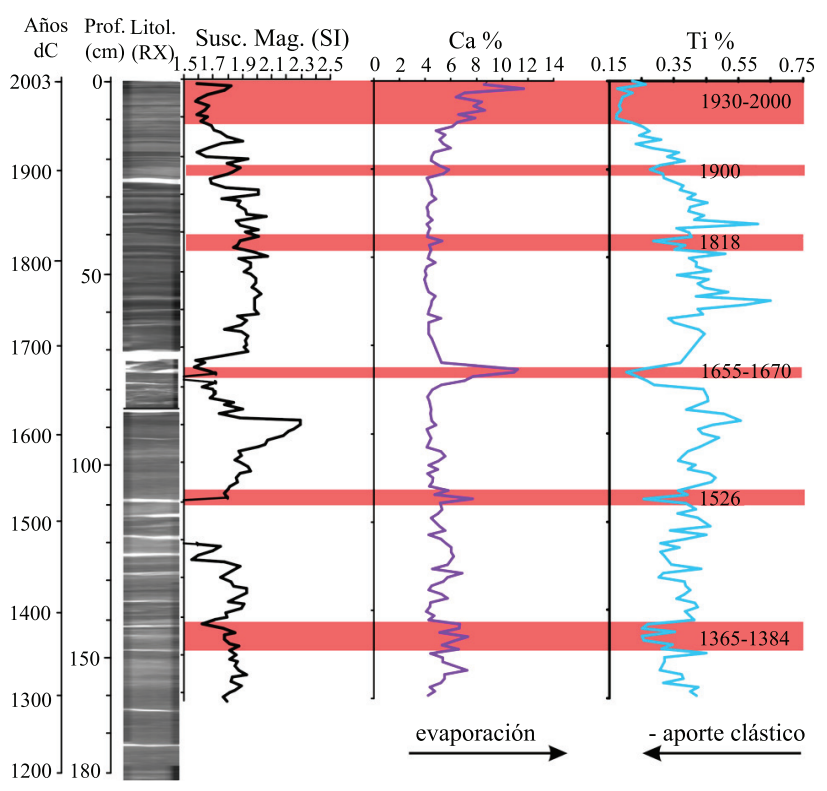

Figura 5. Secuencia estratigráfica de los últimos 700 años del LSMO, con el modelo de edad (años d. C.) al extremo izquierdo. Las radiografías de los sedimentos revelan la estructura laminada de los mismos. Se muestran los datos de la susceptibilidad magnética y concentracines de Ti y Ca. En barras de color se destacan los eventos de sequía más importantes de la secuencia.

las sequías en México (Tabla 2). El conjunto de documentos históricos que aportan datos sobre eventos de sequía incluye los códices aztecas (Escobar, 2004; Therrell et al., 2004), desastres agrícolas asociados a años secos (García-Acosta et al., 2004, Endfield y Fernández Tejedo, 2006) y la relación entre mega-sequías y mega-muertes en el siglo XVI (AcuñaSoto et al., 2002).

Otro conjunto de datos que se toman en consideración en este trabajo son los datos de anillos de árboles que en general son de alta resolución (anual o estacional). El crecimiento anual está influenciado por las condiciones ambientales dominantes durante la formación de los mismos y hay reportes de diferentes partes del centro, norte, noreste y noroeste de México (Díaz et al., 2002; Cleaveland et al., 2003; González-Elizondo et al., 2005; Villanueva-Díaz et al., 2005, 2007, 2009; Cerano-Paredes et al., 2009). Otros estudios documentan la relación entre las sequías históricas y modernas asociadas a la relación entre fenómenos como la Oscilación Multidecadal Atlántica (OMA), el Índice de Oscilación del Sur (IOS) y El Niño para el centro de México y la península de Yucatán (Mendoza et al., 2005, 2007).

5.1. Correlación con datos históricos y de anillos de árboles

Con base en la recopilación de los diferentes registros históricos y de anillos de árboles (Tabla 2) se compara el registro de sequías con el registro de Ti a partir del siglo XIV hasta el siglo XX.

\subsubsection{Siglo $X I V$}

El registro del LSMO muestra para el siglo XIV (Tabla 2, Figura 5) siete valores con baja concentración de Ti que son interpretados como condiciones de menor humedad, destacando los años 1332, 1365-1370, 1380-1384. El año 1332 coincide con el primer reporte histórico de sequía encontrado para el centro de México (año 11 Conejo). Datos provenientes de los códices aztecas señalan la existencia de sequías, plagas y hambre entre 1332 y 1335 (García-Acosta et al., 2004; Therrell et al., 2004).

\subsubsection{Siglo $X V$}

Hay eventos de menor humedad en el LSMO para los años de 1413, 1437, 1442, 1452-1466, 1476 y 1490 que concuerdan con los eventos de sequía reportados en los códices aztecas para los años 1452 a 1455 y con el año 1464 (Tabla 2, Figura 5). El año 1 Conejo (1454) es uno de los más mencionados de la historia azteca ya que fue un evento de gran extensión que causó hambre, enfermedades, escasez, miseria y sequía. Estas condiciones parecen haber iniciado en 1452 (Therrell et al., 2004). Así mismo, 1464 es reportado como un periodo de severas sequías (García-Acosta et al., 2004). Hay datos dendrocronológicos para Nuevo León que abarcan desde 1400 a 1450 (Villanueva-Díaz et al., 2007) y para las áreas de Chihuahua-Sonora y los límites de Sonora, Durango y Sinaloa hay registro de anillos de árboles a partir de 1450 (Tabla 2; Villanueva-Díaz et al., 2009). Con base en estos datos se indica la existencia de eventos secos en Nuevo León de 1412 a 1420 y de 1439 a 1459, mientras que en la región Chihuahua-Sonora se reportan sequías entre 1487-1496. Finalmente, para el área entre Durango, Sonora y Sinaloa hay evidencia de años secos de 1451 a 1455 y de 1462 a 1465.

\subsubsection{Siglo XVI}

Dentro del siglo XVI se registran eventos de menor humedad en el LSMO en los años 1510, 1526, 1536, 1560 y 1569 (Tabla 2; Figura 5). Entre las sequías históricas reportadas coincidentes con las obtenidas para el LSMO se encuentran las sequías de 1524-1525 (García-Acosta et al., 2004) y la denominada mega-sequía de 1540-1570 (Therrell et al., 2006), las cuales, de acuerdo con AcuñaSoto et al. (2002), causaron epidemias y carestía en el centro de México y Yucatán (Mendoza et al., 2005, 2007). Para la cuenca de México, O'Hara y Metcalfe (1997) reportan niveles lacustres bajos durante 1524 y entre 1557 y 1559 . En Nuevo León, los datos de anillos de árboles indican condiciones más secas entre 1516 y 1537. También en el noroeste, para la zona limítrofe entre Durango, Chihuahua y Sinaloa, hay sequías en los intervalos de 1506-1517, 15211524, 1559-1561 y 1566-1573. Asimismo, para la región de Chihuahua-Sonora hay sequías de 1506 a 1515 y de 1559 a 1568 (Villanueva-Díaz et al., 2009).

\subsubsection{Siglo XVII}

Tres eventos de menor humedad se presentan en el 


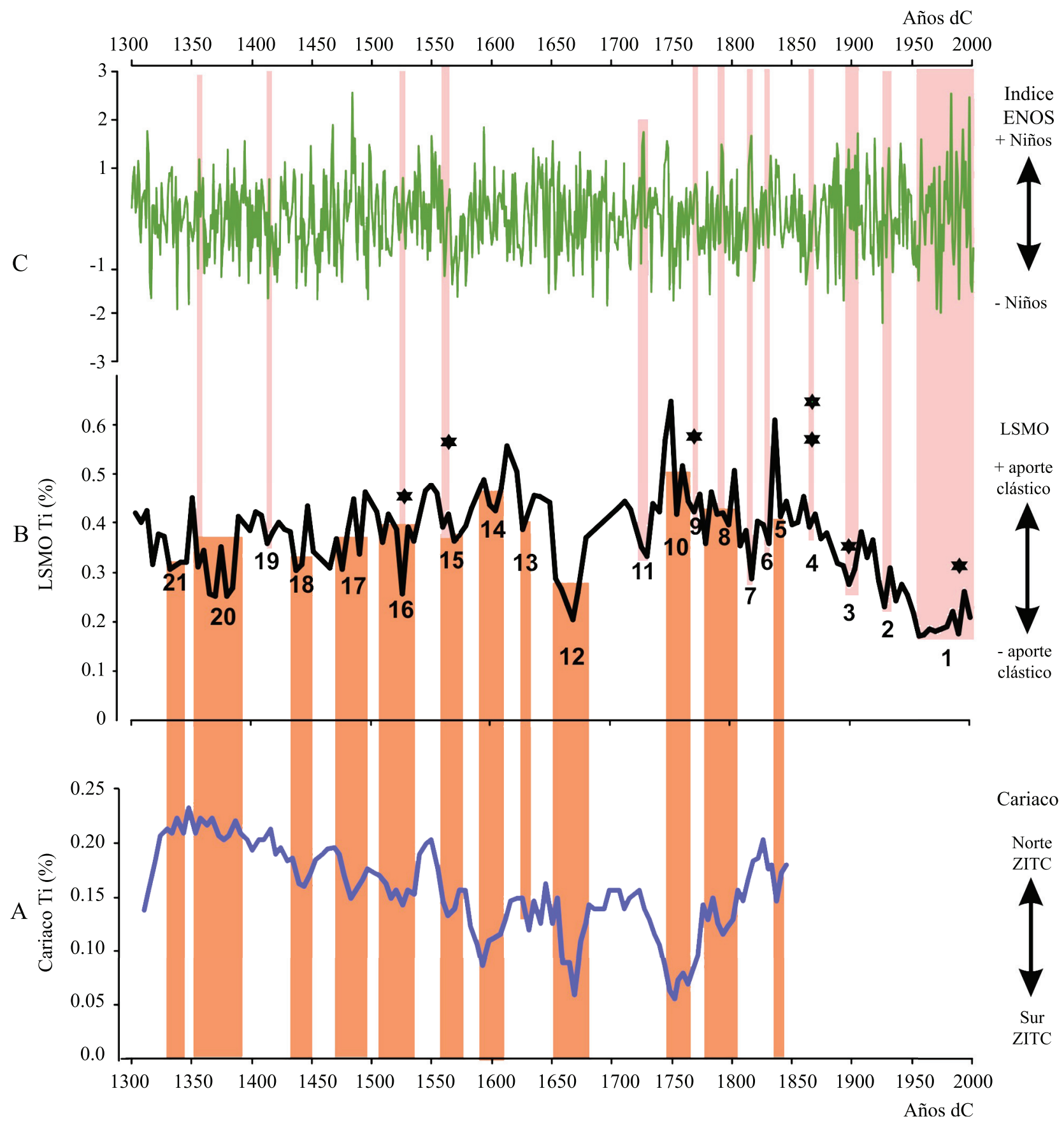

Figura 6. (A) Concentración de Ti (\%) en la cuenca del Cariaco como indicador del desplazamiento latitudinal de la Zona Intertropical de Convergencia (ZITC) (Haug et al., 2001b). (B) Concentración de Ti (\%) del LSMO para los últimos 700 años. Se señalan con barras de color los 21 eventos de sequía que se reconstruyen para el LSMO. (C) Índice de El Niño (Cook et al., 2009). El símbolo de estrella indica eventos de El Niño documentados por otros autores (Gergis y Fowler, 2006; Mendoza et al., 2005) y las barras de color muestran su relación con eventos de sequía del LSMO.

registro del LSMO (Tabla 2; Figura 5): 1601-1605, 1628 y $1655-1670$, siendo este último periodo el evento de menor concentración de todo el registro de Ti. Documentos históricos señalan periodos de falta de lluvias, enfermedades, epidemias (cocolitzi) y hambruna durante 1601, 1604, 1606 y 1624 en el centro de México (Acuña-Soto et al., 2002; García-Acosta et al., 2004; Mendoza et al., 2005). La sequía de 1661 está documentada para la península de Yucatán por
Mendoza et al. (2007), y en el sureste de Coahuila hay un periodo intenso de sequía de 1662-1670 (Constante García, et al., 2009).

Para el noreste los datos dendrocronológicos indican sequías durante 1629-1632 y 1659- 1670 (Villanueva-Díaz et al., 2007). En la región de Durango, Chihuahua y Sinaloa se han detectado sequías durante los periodos 1559-1602, $1604-1606,1622-1628,1662-1664$ y $1666-1669$, mientras 
60

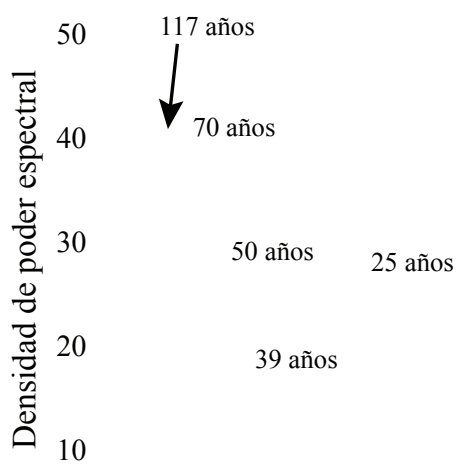

0.00

0.05

Frecuencia (1/año)

Figura 7. Ciclicidad del registro de Ti en el que se observan periodicidades de 25, 39, 50, 70 y 117 años en el intervalo analizado de 1300-2000.

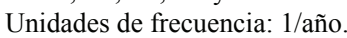

que en Chihuahua y Sonora se han registrado para 16221626 y 1666-1670 (Villanueva-Díaz et al., 2009). Para el río Yaqui se detecta una sequía entre 1662 y 1678 (Nicholas y Battisti, 2008).

\subsubsection{Siglo XVIII}

En el registros del LSMO (Tabla 2; Figura 5), se encontraron siete valores bajos de Ti que sugieren menor humedad: 1731, 1741, 1756, 1770, 17801789 y 1799. Éstos concuerdan con las sequías históricas reportadas para el centro de México en 1731, 1741, 1756, 1770, 1780, 1789 y 1799 (Garza-Merodio, 2002; García-Acosta et al., 2004). Para Chihuahua, Endfield y Fernández Tejedo (2006) documentan sequías para los años 1739-41, 1755, 1770-1773.

La comparación de los datos del LSMO con la reconstrucción de precipitación de invierno-primavera a partir de anillos de árboles en Chihuahua muestra coincidencia con el año de 1742 (Díaz et al., 2002). En Nuevo León hay sequías detectadas para el periodo de 1784-1789, y en Durango, Chihuahua y Sinaloa de 1750 a 1768. Para Chihuahua-Sonora los periodos secos ocurrieron en 1732-1734, 1752-1761, 1770-1779, 1797-1806. Para la cuenca del río Yaqui los periodos que coinciden con el LSMO son 1728-1733, 1772-1782 y 1785-1790 (Nicholas y Battisti, 2008).

\subsubsection{Siglos $X I X Y X X$}

Para el siglo XIX (Tabla 2; Figura 5), el registro de Ti presenta nueve eventos de menor aporte clástico: 1808, $1818,1832,1842,1851-1857,1866,1876$ y 1890 . La correspondencia entre la edad de los eventos en el registro de Ti y las documentadas en los registros históricos de
México es de más del 60\% (Tabla 2). Los valores de concentración de Ti presentan una tendencia a disminuir a partir de 1860. Para el siglo XX se documentan en el LSMO periodos secos con bajos valores de Ti durante 1900, 1914, 1929, 1939, 1958, 1981 y 1991 (Tabla 2; Figura 5). Hay evidentemente una mayor disponibilidad de reportes históricos y datos dendrocronológicos para los siglos XIX y XX, lo que permite comparar con mayor precisión los eventos secos (Tabla 2).

La correspondencia temporal entre las sequías reportadas a través de los registros históricos y de anillos de árboles en varias zonas del país con las detectadas en el LSMO sugiere que el Ti es un indicador adecuado para reconstruir eventos de baja precipitación. La utilización de este indicador de alta resolución abre la posibilidad de identificar, con mayor precisión que en trabajos anteriores, eventos de sequía en periodos que carezcan de registros históricos, dendrocronológicos o instrumentales.

Gran parte del registro de Ti del LSMO abarca el periodo denominado Pequeña Edad de Hielo (1350-1850), que en otras regiones de México ha sido documentado como una periodo de gran variabilidad climática (Hodell et al., 2005; Lozano-García et al., 2007). Los datos de concentración de Ti muestran también dicha variabilidad, con fluctuaciones entre eventos de sequía y de humedad para la región occidental del país, donde se localiza el LSMO. A partir de 1845 hay una clara tendencia de disminución en la concentración de Ti en el registro.

Para estimar si hay correspondencia entre los datos de Ti reconstruidos con datos de precipitación registrada instrumentalmente, se buscaron los datos disponibles de precipitación anual de la estación meteorológica de Santa María del Oro. Sin embargo, el registro disponible solo abarca desde 1965 y está incompleto, faltando varios años. Otra estación cercana al lago que presenta un registro climático largo es la de Ahuacatlán, con datos desde el año 1935. La comparación de los datos de precipitación anual de esta estación con el registro de Ti (\%) desde 1935 muestra cierta correspondencia (Figura 8), aunque cabe señalar que los datos de Ti no son anuales a diferencia de los datos de precipitación. No obstante, sí se observan las tendencias entre ambos registros, indicando cierto grado de similitud.

\subsection{Ciclicidad y posibles mecanismos de cambio climático}

El análisis espectral de los datos de concentración de Ti en el intervalo de 1300 a 2000 sugiere la existencia de sequías recurrentes en diferentes periodicidades: 25,39 , 50, 70 y 117 años (Figura 7). En otros registros de México se han descrito ciclicidades para explicar fases de cambio climático. Por ejemplo, periodicidades de 11, 22 y 45 años han sido referidas para el oeste de México (latitudes $19^{\circ} \mathrm{N}$ a $32^{\circ} \mathrm{N}$ ), relacionándolas con el fenómeno de El Niño y la actividad solar, sugiriendo que ambos fenómenos modifican la circulación a gran escala y aumentan el desarrollo de 
Tabla 2. Compilación de información sobre sequías históricas proveniente de documentos históricos y de anillos de árboles reportadas por diferentes autores.

\begin{tabular}{|c|c|c|c|c|c|c|c|c|c|c|}
\hline LSMO, Nayarit & $\begin{array}{c}\text { Baja California } \\
\text { Sur }\end{array}$ & Saltillo & Nuevo León & $\begin{array}{l}\text { Durango, } \\
\text { Chihuahua }\end{array}$ & $\begin{array}{l}\text { Límitie de } \\
\text { Chihuahua, } \\
\text { Sonora }\end{array}$ & Río Yaqui & $\begin{array}{l}\text { Durango, } \\
\text { Zacatecas }\end{array}$ & Coahuila & Chihuahua & Chihuahua \\
\hline Este registro & $\begin{array}{l}\text { Díaz et al ., } \\
2001\end{array}$ & $\begin{array}{c}\text { Villanueva et } \\
\text { al., } 2007\end{array}$ & $\begin{array}{c}\text { Villanueva et } \\
\text { al., } 2007\end{array}$ & $\begin{array}{c}\text { Villanueva et } \\
\text { al., } 2009\end{array}$ & $\begin{array}{c}\text { Villanueva et } \\
\text { al., } 2009\end{array}$ & $\begin{array}{c}\text { Nicholas y } \\
\text { Battisti, } 2008\end{array}$ & $\begin{array}{l}\text { González- } \\
\text { Elizondo et al ., } \\
2005\end{array}$ & $\begin{array}{l}\text { Constante- } \\
\text { García et al ., } \\
2009\end{array}$ & $\begin{array}{c}\text { Cerano-Paredes } \\
\text { et al ., } 2009\end{array}$ & $\begin{array}{l}\text { Díaz et al ., } \\
2002\end{array}$ \\
\hline $\mathrm{Ti}$ & Anillos árboles & Anillos árboles & Anillos árboles & Anillos árboles & Anillos árboles & Anillos árboles & Anillos árboles & Anillos árboles & Anillos árboles & Anillos árboles \\
\hline \multicolumn{11}{|l|}{ Siglo XIV } \\
\hline \multicolumn{11}{|l|}{1332} \\
\hline \multicolumn{11}{|l|}{1341} \\
\hline \multicolumn{11}{|l|}{1365} \\
\hline \multicolumn{11}{|l|}{1370} \\
\hline \multicolumn{11}{|l|}{1380,1384} \\
\hline \multicolumn{11}{|l|}{1398} \\
\hline \multicolumn{11}{|l|}{ Siglo XV } \\
\hline 1413 & & & $1412-1420$ & & & & & & & \\
\hline \multicolumn{11}{|l|}{1437} \\
\hline 1442 & & & $1439-1459$ & & & & & & & \\
\hline 1452 & & & & $1451-1455$ & & & & & & \\
\hline 1466 & & & & $1462-1465$ & & & & & & \\
\hline 1476 & & & & $1473-1476$ & & & & & & \\
\hline 1490 & & & & & $1487-1496$ & & & & & \\
\hline \multicolumn{11}{|l|}{ Siglo XVI } \\
\hline 1510 & & & & $1506-1517$ & $1506-1515$ & & & & & \\
\hline 1526 & & & $1516-1537$ & $1521-1524$ & & & & & & \\
\hline \multicolumn{11}{|l|}{1536} \\
\hline 1560 & & & $1559-1590$ & $1559-1561$ & $1552-1573$ & & & & & \\
\hline 1569 & & & & $1566-1573$ & & & & & & \\
\hline \multicolumn{11}{|l|}{ Siglo XVII } \\
\hline $1601-1605$ & & & & $\begin{array}{l}1599-1602 \\
1604-1606\end{array}$ & & & & $1595-1618$ & & \\
\hline 1628 & & & $1629-1632$ & $1622-1628$ & $1611-1626$ & & & & & \\
\hline 1655 & & & & & $1644-1654$ & & & & & \\
\hline 1670 & & $1659-1670$ & & 1669 & $1666-1670$ & $1662-1678$ & & $1662-1670$ & & \\
\hline \multicolumn{11}{|l|}{ Siglo XVIII } \\
\hline 1731 & & & & & $1732-1734$ & $1728-1733$ & & $1730-1743$ & & \\
\hline 1741 & & & $1738-1743$ & & & & $1739-1743$ & & & 1742 \\
\hline 1756 & & & & $1750-1768$ & $1751-1756$ & & $1749-1755$ & $1750-1756$ & $1753-1760$ & $1751-1765$ \\
\hline 1770 & & & & & $1767-1779$ & & $1772-1779$ & & & \\
\hline 1780 & & & & & & $1772-1782$ & & & & \\
\hline 1789 & & & $1784-1790$ & & & $1785-1790$ & & $1784-1794$ & $1785-1792$ & \\
\hline 1799 & & & & $1797-1805$ & $1797-1806$ & & $1797-1805$ & & $1798-1806$ & $1798-1810$ \\
\hline \multicolumn{11}{|l|}{ Siglo XIX } \\
\hline 1808 & & $1806-1813$ & $1806-1809$ & & & & & $1807-1812$ & & \\
\hline 1818 & & & $1817-1820$ & & $1817-1826$ & & & & $1819-1830$ & \\
\hline 1832 & & & & & $1830-1832$ & & & $1819-1832$ & & 1820 \\
\hline 1842 & & & & $1847-1850$ & & & & & $1841-1870$ & \\
\hline $1851-1857$ & & & $1857-1868$ & & $1855-1864$ & $1857-1881$ & $1857-1876$ & $1850-1857$ & & \\
\hline 1866 & & & $1867-1875$ & $1869-1868$ & & & & $1860-1884$ & & \\
\hline 1876 & & $1870-1875$ & & & & & & & & \\
\hline 1890 & & $1889-1897$ & & $1982-1894$ & & & & $1891-1896$ & $1890-1897$ & $1880-1896$ \\
\hline \multicolumn{11}{|l|}{ Siglo XX } \\
\hline 1900 & 1905 & & $1907-1910$ & & & & $1907-1910$ & & & \\
\hline 1914 & 1912 & & $1916-1917$ & & & & $1916-1922$ & & & \\
\hline 1929 & & & $1927-1933$ & & & & & $1917-1934$ & & \\
\hline 1939 & $1939-1958$ & & & $1934-1939$ & $1934-1943$ & & $1932-1939$ & & $1924-1941$ & $1939-1958$ \\
\hline 1958 & 1957 & $1952-1956$ & & $1950-1959$ & $1945-1960$ & $1950-1957$ & & $1945-1962$ & $1952-1957$ & 1954 \\
\hline 1981 & 1983 & & $1979-1985$ & & & & & & & 1980 \\
\hline 1991 & & & & $1993-2000$ & $1993-2002$ & & & $1993-2004$ & $1994-2005$ & \\
\hline
\end{tabular}


Tabla 2. Compilación de información sobre sequías históricas proveniente de documentos históricos y de anillos de árboles reportadas por diferentes autores.

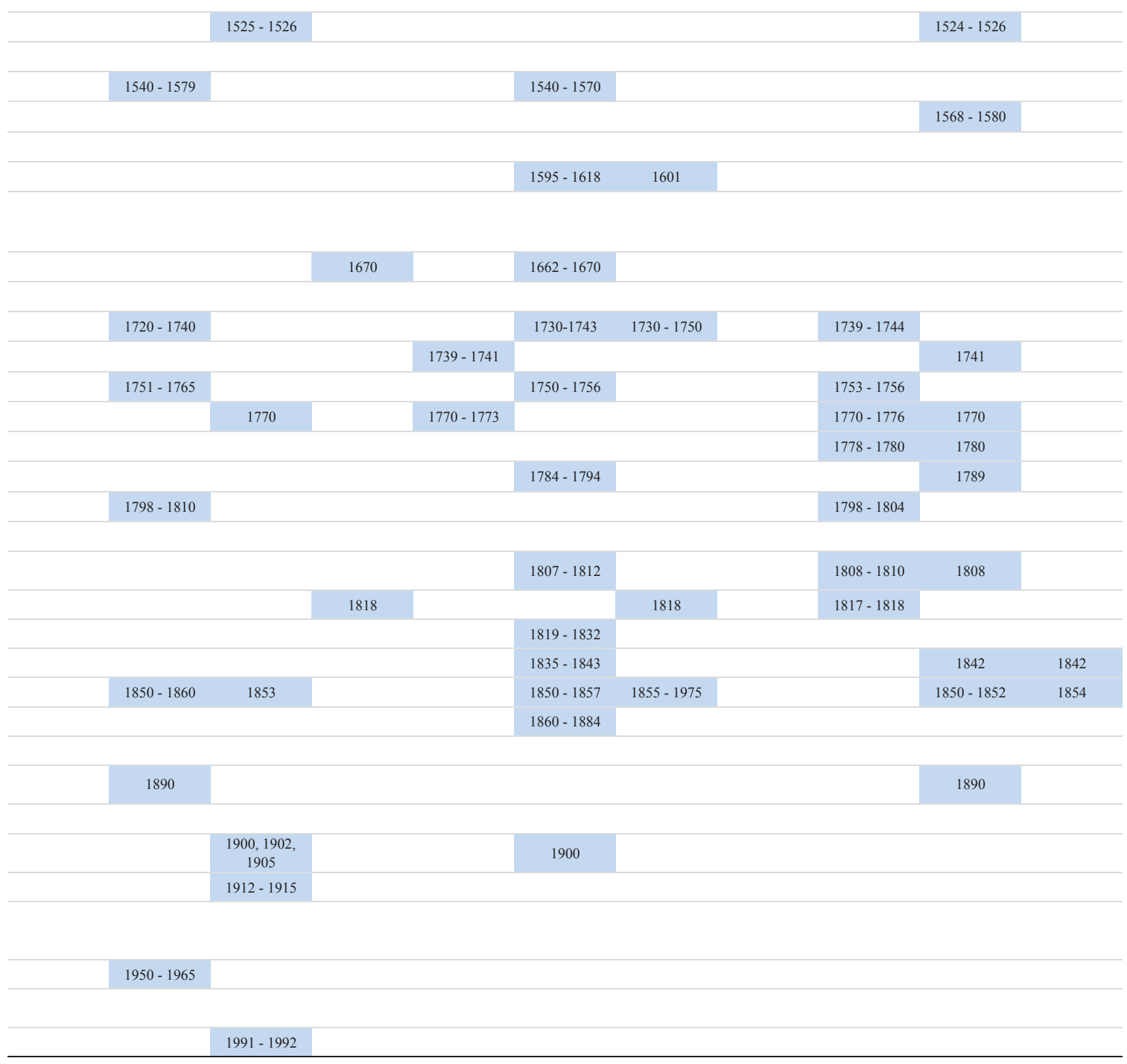




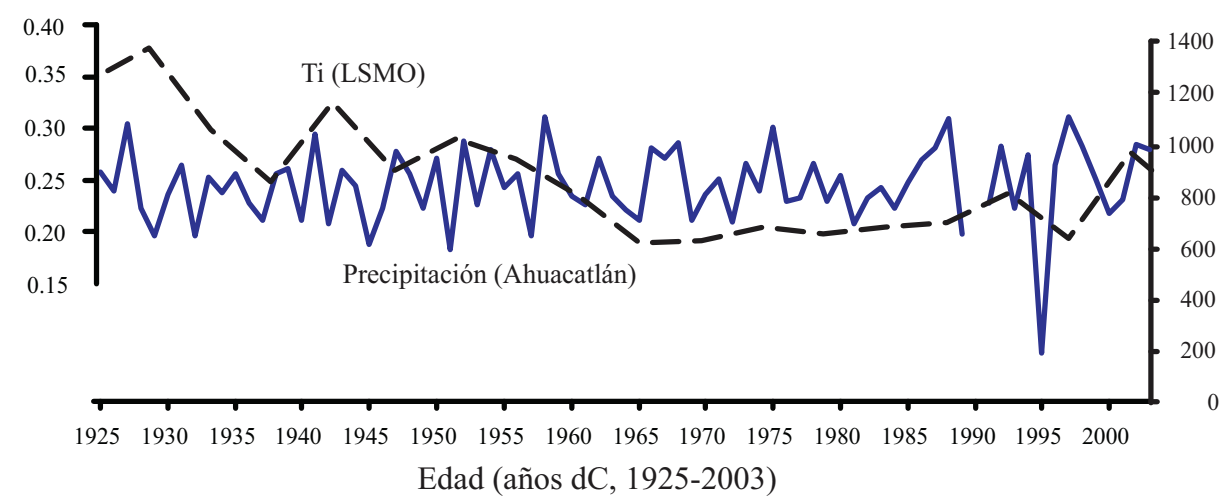

Figura 8. Comparación de los datos de precipitación anual de la estación climática de Ahuacatlán (21³'18” N, 104²9’3” W) con el registro de Ti del LSMO desde 1925 hasta 2003.

fuertes anomalías climáticas con respecto a la temperatura y la precipitación (Tereshchenko et al., 2003). Mendoza et al. (2005) documentan periodicidades de 22, 24, 32, 39 y 89 años en el centro de México e indican que picos de 39 años pueden estar asociados con eventos de El Niño. Para el área maya, Mendoza et al., (2007) reconocen periodicidades de $\sim 40$ años que relacionan con la Oscilación Multidecadal Atlántica (AMO). Otras ciclicidades reconocidas en Yucatán son 24-27, 39 y 50 años (Hodell et al., 2001, 2005).

La variabilidad climática en escalas de décadas y centurias asociada al forzamiento solar ha sido discutida en otros registros, tanto lacustres como marinos. Sin embargo, los mecanismos climáticos involucrados que subyacen a este proceso no están claramente establecidos. Uno de los mecanismos que se ha sugerido para explicar las sequías de 1350 a 1850 en Yucatán y en la cuenca del Cariaco (Venezuela), es el desplazamiento de la Zona Intertropical de Convergencia (ZITC) a una posición promedio más al sur. Esto reduce los vientos del este que abastecen los vientos húmedos del Caribe durante el verano (Haug et al., 2001a; Hodell et al., 2005), causando un decremento en las lluvias de verano. Este mecanismo, que tuvo consecuencias en la región de Yucatán, pudo afectar también gran parte de México ya que la mayor parte de la lluvia en el país ocurre en el verano. El desplazamiento hacia el sur de la ZITC resulta en una menor actividad convectiva cerca de México y, consecuentemente, en una menor cantidad de lluvias (Magaña et al., 1999).

En la Figura 6 se muestra la relación temporal entre los eventos de sequía documentados a través del registro de Ti del LSMO, el registro del aporte de Ti a la cuenca del Cariaco (Haug et al., 2001b), el cual puede ser considerado como un indicador de la posición media de la ZCIT, y con el índice de El Niño para los últimos siete siglos (Cook et al., 2009). Hay una relación entre los eventos secos observados en el LSMO (eventos 5, 8, 10, 12, 13, 14, 15, 16, 17, 18, 20 y 21; Tabla 3) y la disminución en los valores de Ti de la cuenca del Cariaco.

Los eventos $1,2,3,4,6,7,9,11$ y 19 muestran la correspondencia entre la reducción en la concentración de Ti y los eventos de El Niño, los cuales no siempre son desencadenados al desplazarse la ZITC hacia el sur, ya que al parecer necesitan además de otros mecanismos para dispararse como se puede observar en los eventos 11 y 19 de la gráfica de Ti (Figura 6B). Algunos de estos eventos de El Niño, pueden afectar el monzón, debilitándolo y adelantándolo o atrasándolo. Lo anterior puede significar una temporada de lluvias deficientes (Higgins et al., 1997), causando sequías más intensas, como las que se sugieren para los eventos 15 y 8 y las que se presentan en el evento 1 , donde se observa un incremento en el número de eventos El Niño y una reducción importante en el aporte de Ti al LSMO. Eventos de El Niño que han sido reportados por otros autores (Mendoza et al., 2005; Gergis y Fowler, 2006) se observan en el registro de Ti de LSMO (eventos $4,9,15$ y 16) con bajos porcentajes de Ti (Figura 6B). Hay otros eventos de menor humedad donde se documenta una combinación de los mecanismos que pueden causar sequías, una posición al sur de la ZITC y la presencia de El Niño como ocurre en los eventos 8, 15, 16 y 20.

\section{Conclusiones}

El análisis de la concentración elemental en secuencias sedimentarias lacustres a través de la utilización del ITRX provee datos de alta resolución para evaluar la variabilidad climática. Los datos así obtenidos ofrecen amplias posibilidades en el estudio del cambio ambiental en las zonas tropicales donde hay escasos registros de alta resolución.

El registro de Ti del LSMO sugiere la existencia de 21 eventos de sequía los cuales han sido validados comparándolos con el registro histórico y con datos de anillos de árboles. Entre los 21 eventos de sequía, hay seis que destacan en el registro por sus bajos valores de Ti: el de 1365 a 1384, referido en códices aztecas como años de hambruna y calamidades; el evento de 1526, registrado en anillos de árboles de la región de Nuevo León; el periodo de 1655 a 1670, para el que se registran valores muy bajos 
Tabla 3. Edades de los eventos de sequía señalados en la Figura 6 y su relación con factores climáticos.

ZITC $=$ Zona Intertropical de Convergencia

de Ti, está reportado en amplias zonas del norte y sólo es comparable en sus valores a lo registrado para el siglo XX; los tres eventos restantes, 1818,1900 y 1930-2000, han sido reportados tanto en documentos escritos como en anillos de árboles. Los resultados de este trabajo muestran la utilidad del uso de datos geoquímicos para establecer con mayor resolución el cambio ambiental y en este caso el uso del registro de Ti para inferir cambios en el aporte clástico y, por lo tanto, en la humedad. Pero lo más importante es la resolución que alcanzan estos datos, ya que los resultados de estudios paleoecológicos en otras secuencias lacustres del centro de México con indicadores como diatomeas, polen, etc., sólo indican tendencias generales de cambio climático.

Los mecanismos causales de los eventos de sequía en el extremo occidental de México están relacionados tanto a la actividad solar como a otros factores, como la posición que alcanza durante el verano la ZITC, y la disminución de la intensidad del Monzón Mexicano por eventos como El Niño. Sin embargo, falta explorar y profundizar en las interacciones de estos factores climáticos que determinan la variabilidad climática en la región tropical.

En general se puede concluir que el Ti funciona como un indicador de menor aporte clástico y precipitación en sistemas lacustres similares al LSMO. Su uso para el estudio del cambio climático abre la posibilidad de registrar con mayor resolución la variabilidad climática para edades previas al registro histórico e instrumental aportando datos sobre el funcionamiento del sistema climático en épocas pasadas.

\section{Agradecimientos}

Agradecemos al grupo del Limnological Research Center de la Universidad de Minnesota por su apoyo en la perforación y las facilidades en la documentación y la descripción de los núcleos, en particular a D. Schnurrenberger, A. Noren y M. Shapley a través del proyecto "Mexican Lakes: MOLE". También queremos expresar nuestro agradecimiento a los colegas de la Universidad de Aberystwyth y Nottingham por el apoyo durante el trabajo de perforación y a Matthew Jones por su apoyo con el escáner ITRAX. El apoyo y la participación de la Dra. Isabel Israde y de la Dra. Beatriz Ortega fueron fundamentales durante el proceso de muestreo. Este trabajo recibió apoyo financiero de los proyectos DGAPA IN 203102 y IN110106, "Estudio paleoambiental de alta resolución en el Lago Santa María del Oro, Nayarit". Agradecemos también el apoyo del Quím. Rufino Lozano en el análisis de XRF.

\section{Referencias}

Acuña-Soto, R., Sthale, D.W., Cleaveland, M.K., Therrell, M.D., 2002, Megadrought and megadeath in $16^{\text {th }}$ Century Mexico: Revista Biomédica, 13, 289-292.

Adams, D.K., Comrie, A.C., 1997, The North American Monsoon: Bulletin of the American Meteorological Society, 78, 2197-2213.

Armienta, M.A., Vilaclara, G., De la Cruz-Reyna, S., Ramos, S., Ceniceros, N., Cruz, O., Aguayo, A., Arcega-Cabrera, F., 2008, Water chemistry of lakes related to active and inactive Mexican volcanoes: Journal of Volcanology and Geothermal Research., 178, 249-258.

Barron, J.A., Bukry, D., Bischoff, L.L, 2003, A 2000-yr-long record of climate from the Gulf of California, in West, G.J., Blomquist, N.L. (eds.), Proceedings of the Nineteenth Pacific Climate Workshop, Asilomar, Pacific Grove, California, March 3-6, 2002. Technical Report 71: Sacramento, California, Interagency Ecological Program for the San Francisco Estuary, 11-21.

Barron, J.A., Bukry, D., Bischoff, L.L., 2004, High resolution paleoceanography of the Guaymas Basin, Gulf of California, during the past 15000 years: Marine Micropaleontology, 50, 185-207.

Boyle, J.F., 2001, Inorganic geochemical methods in paleolimnology, in Last, W.M., Smol, J.P. (eds.), Tracking Environmental Change Using Lake Sediments, Volume 2, Physical and Geochemical Methods: Dordrecht, Países Bajos, Kluwer Academic Publishers, 83-141.

Bradbury, J.P., 2000, Limnologic history of Lago de Pátzcuaro, Michoacán, Mexico for the past 48000 years: impacts of climate and man: Palaeogeography, Paleoclimatology, Palaeoecology, 163, 69-95.

Caballero, M., Ortega-Guerrero, B., 1998, Lake levels since about 40000 years ago lake at Lake Chalco, near Mexico City: Quaternary Research, 50, 69-79.

Caballero, M., Lozano, S., Ortega, B., Urrutia, J., Macias, J.L., 1999, Environmental characteristics of Lake Tecocomulco, northern basin of Mexico, for the last 50000 years: Journal of Paleolimnology, 22, 399-411.

Caballero, M., Vázquez, G., Lozano-García, S., Rodríguez, A., SosaNájera, S., Ruíz- Fernández, A.C., Ortega, B., 2006, Present limnological conditions and recent ( $c a .340 \mathrm{yr}$ ) palaeolimnology of a tropical lake in the Sierra de los Tuxtlas, eastern Mexico: Journal of Palaeolimnology, 35, 83-97. 
Cerano-Paredes, J., Villanueva-Díaz, J. Arreola-Ávila, J.G., Sánchez-Cohen, I., Valdez-Cepeda, R., García-Herrera, G., 2009, Reconstrucción de 350 años de precipitación para el suroeste de Chihuahua, México: Madera y Bosques, 15, 27-44.

Cleaveland, M.K., Stahle, D.W., Therrell, M.D., Villanueva-Díaz, J., Burns, B.T., 2003, Tree ring reconstructed winter precipitation in Durango, Mexico: Climatic Change, 59, 369-388.

Constante García, V., Villanueva Díaz, J., Cerano Paredes, J., Cornejo Oviedo, E.H., Valencia Manzo, S., 2009, Dendrocronología de Pinus cembroides Zucc. y reconstrucción de precipitación estacional para el sureste de Coahuila: Revista Ciencia Forestal en México, 34, 17-38.

Cook, E.R., D'Arrigo, R.D., Anchukaitis, K.J., 2009, Tree ring 500 year ENSO Index reconstructions (base de datos): Boulder, Colorado, EE.UU., World Data Center for Paleoclimatology/NOAA Paleoclimatology Program, Data Contribution Series \# 2009-105.

Croudace, I.W., Rindby, A., Rothwell, R.G., 2006, ITRAX: description and evaluation of a new multi-function X-ray core scanner, in Rotwell, R.G. (ed.), New Techniques in Sediment Core Analysis (Special Publication No. 267): Londres, The Geological Society, 51-64.

Curtis, J.H., Hodell, D.A., Brenner, M., 1996, Climate variability on Yucatan Peninsula (Mexico) during the past 3500 years, and implications for Maya cultural evolution: Quaternary Research, 46, 37-47.

Díaz, S.C., Touchan, R., Swetnam, T.W., 2001, A tree-ring reconstruction for Baja California Sur, Mexico: International Journal of Climatology, 21, 1007-1009.

Díaz, S.C., Therrell, M.D., Stahle, D.W., Cleaveland, M.K., 2002, Chihuahua (Mexico) winter-spring precipitation reconstructed from tree-rings, 1647-1992: Climate Research, 22, 237-244.

Endfield, G.H., Fernández Tejedo, I., 2006, Decades of drought, years of hunger: Archival investigations of multiple year droughts in late colonial Chihuahua: Climatic Change, 75, 391-419.

Escobar, A., 2004, Desastres agrícolas en México. Catálogo histórico II Siglo XIX (1822-1900): México, D. F., Fondo de Cultura Económica, $280 \mathrm{p}$.

Eugster, H.P., 1980, Geochemistry of evaporitic lacustrine deposits: Annual Review of Earth and Planetary Sciences, 8, 35-63.

Ferrari, L., Nelson, S.A., Rosas-Elguera, J., Aguirre, G., Venegas, S., 1997, Tectonics and volcanism of the western Mexican Volcanic Belt, in Aguirre, G., Aranda, J., Carrasco, G., Ferrari, L. (eds.), Magmatism and tectonics in the central and northwestern Mexico - a selection of the 1997 IAVCEI General Assembly excursions: México, D. F., Universidad Nacional Autónoma de México, Instituto de Geología, 85-129.

Figueroa-Rangel, B.L., Willis, K.J., Olvera-Vargas, M., 2008, 4200 years of Pine-dominated upland forest dynamics in west-central Mexico: human or natural legacy?: Ecology, 89, 1893-1907.

Filonov, A, Tereshchenko, I., Vargas, A.C., 2005, Resultados del estudio del régimen termodinámico del lago volcánico Santa Maria del Oro, Nayarit: Geos, 25, 139-140.

García-Acosta, V., Pérez Zevallos, J.M., Molina del Villar, A., 2004, Desastres agrícolas en México. Catálogo histórico, I. Épocas prehispánica y colonial (958-1822): México, D. F., Fondo de Cultura Económica, 506 p.

Garza-Merodio, G.G., 2002, Frecuencia y duración de sequías en la cuenca de México de fines del siglo XVI a mediados del XIX: Boletín del Instituto de Geografía UNAM, 48, 106-115.

Gergis, J.L., Fowler, A.M., 2006, How unusual was late 20th century El Niño-Southern Oscillation (ENSO)? Assessing evidence from tree-ring, coral, ice-core and documentary palaeoarchives, A.D. 1525-2002: Advances in Geosciences, 6, 173-179.

González-Elizondo, M., Jurado, E., Návar, J., González-Elizondo, M.S., Villanueva, J., Aguirre, O., Jiménez, J., 2005, Tree rings and climate relationships for Douglas-fir chronologies from the Sierra Madre Occidental, Mexico: A 1681-2001 rain reconstruction: Forest Ecology and Management, 213, 39-53.

Haug, G.H., Haughen, K.A., Sigman, D.S., Peterson, L.C., Röhl, U., 2001a, Southward migration of the Intertropical Convergence Zone through the Holocene: Science, 293, 1304-1308.
Haug, G.H., Haughen, K. A., Sigman, D.S., Peterson, L.C. Röhl, U., 2001b, Cariaco Basin Trace Metal Data (base de datos): Boulder, Colorado, E.U.A., World Data Center for Paleoclimatology/NOAA Paleoclimatology Program, Data Contribution Series \#2001-071.

Haug, G.H., Günther, D., Peterson, L.C., Sigman, D.S., Haughen, K.A., Aeschlimann, B., 2003, Climate and collapse of Maya civilization: Science, 299, 1731-1735.

Higgins, R.W., Yao, Y., Wang, X.L., 1997, Influence of the North American monsoon system on the U.S. summer precipitation regime: Journal of Climate, 10, 2600-2622.

Hodell, D.A., Brenner, M., Curtis, J.H., Guilderson, T., 2001, Solar forcing of drought frequency in the Maya lowlands: Science, 292, 1367-1370.

Hodell, D.A., Brenner, M., Curtis, J.H., Medina-González, R., Chan Can, E.I., Albornaz-Pat, A., Guilderson, T.P., 2005, Climate change on the Yucatán Peninsula during the Little Ice Age: Quaternary Research, 63, 109-121

Israde-Alcántara, I., Garduño-Monroy, V.H, Ortega, M.R., 2002, Paleoambiente lacustre del Cuaternario tardío en el centro del lago de Cuitzeo: Hidrobiologica, 12, 61-78.

Kelts, K., Hsü, K.J., 1978, Freshwater carbonate sedimentation, in Lerman, A. (ed.), Lakes - Chemistry, Geology, Physics: Nueva York, Springer-Verlag, 295-323.

Kienel, U., Bowen, W.S., Byrne, R., Park, J., Böhnel, H., Dulski, P., Luhr, J.F., Siebert, L., Haug, G.H., Negendank, J.F.W., 2009, First lacustrine varve chronologies from Mexico: impact of droughts, ENSO and human activity since AD 1840 as recorded in maar sediments from Valle de Santiago: Journal of Paleolimnology, 42, 587-609.

Lozano-García, M.S., Ortega-Guerrero, B., Sosa-Nájera, S., 2002, Mid- to late-Wisconsin pollen record of San Felipe Basin, Baja California: Quaternary Research, 58, 84-92.

Lozano-García, S, Caballero, M., Ortega, B., Rodríguez, A., Sosa, S., 2007, Tracing the effects of the Little Ice Age in the tropical lowlands of eastern Mesoamerica: Proceedings of the National Academy of Science, 104, 16200-16203.

Magaña, V.O., Amador, J.A., Medina, S., 1999, The midsummer drought over Mexico and Central America: Journal of Climate, 12, 15771588.

Magaña, V.O., Vázquez, J.L., Pérez, J.L., Pérez, J.B., 2003, Impact of El Niño on precipitation in Mexico: Geofísica Internacional, 42, 313-330.

MathWorks, 2004, MATLAB 7 (programa informático, CD-ROM): Natick, Massachusetts, E.U.A., The MathWorks, 1 CD-ROM.

Méndez, M., Magaña, V.O., 2010, Regional aspects of prolonged meteorological droughts over Mexico and Central America: Journal of Climate, 23, 1175-1188.

Mendoza, B., Jauregui, E., Díaz-Sandoval, R., García-Acosta, V., Velasco, V., Cordero, G., 2005, Historical droughts in Central México and their relation with El Niño: Journal of Applied Meteorology, 44, 709-716.

Mendoza, B., García-Acosta, V., Velasco, V., Jauregui, E., Díaz-Sandoval, R., 2007, Frequency and duration of historical droughts from the 16th to the $19^{\text {th }}$ centuries in the Mexican Maya lands, Yucatan Peninsula: Climatic Change, 83, 151-168.

Metcalfe, S.E., 1995, Holocene environmental change in the Zacapu Basin, Mexico: a diatom-based record: The Holocene, 5, 196-208.

Metcalfe, S.E., Bimpson, A., Courtice, A.J., O'Hara, S.L., Taylor, D.M., 1997, Climate change at the monsoon/westerly boundary in Northern Mexico: Journal of Paleolimnology, 17, 155-171.

Nelson, S.A., Sánchez-Rubio, G., 1986, Trans Mexican Volcanic Belt Field Guide: México. D.F., México, Geological Association of Canada, Volcanology Division, Universidad Nacional Autónoma de México, 108 p.

Nicholas, R.E., Battisti, D.S., 2008, Drought recurrence and seasonal rainfall prediction in the Río Yaqui Basin, Mexico: Journal of Applied Meteorology and Climatology, 47, 991-1005.

O'Hara, S.L., Metcalfe, S.E., 1997, The climate of Mexico since the Aztec period: Quaternary International 43/44, 25-31. 
O'Sullivan, P.E., 1983, Annually-laminated lake sediments and the study of Quaternary environmental changes - a review: Quaternary Science Reviews, 1, 245-313.

Ortega-Guerrero, B., Thompson, R., Urrutia Fucugauchi, J., 2000, Magnetic properties of lake sediments from Lake Chalco, central Mexico, and their palaeoenvironmental implications: Journal of Quaternary Science, 15, 127-140.

Ortega-Rosas, C.I., Peñalba, M.C., Guiot, J., 2008, Holocene altitudinal shifts in vegetation belts and environmental changes in the Sierra Madre Occidental, Northwestern México, based on modern and fossil pollen data: Review of Palaeobotany and Palynology, 151, $1-20$.

Panel Intergubernamental de Cambio Climático (IPCC), 2007, Informe del Grupo de Trabajo I - Base de las Ciencias Físicas, Solomon, S., Qin, D., Manning, M., Chen, Z., Marquis, M., Averyt, K.B., Tignor, M., Miller, H.L. (eds.): Cambridge, Reino Unido y Nueva York, E.U.A., 996 p.

Pérez-Cruz, L., 2006, Climate and ocean variability during the middle and late Holocene recorded in laminated sediments from Alfonso Basin, Gulf of California, Mexico: Quaternary Research, 65, 401-410.

Peterson, L.C., Haug, G.H., 2006, Variability in the mean latitude of the Atlantic Intertropical Convergence Zone as recorded by riverine input of sediments to the Cariaco Basin (Venezuela): Palaeogeography, Palaeoclimatology, Palaeoecology, 234, 97-113.

Peterson, L.C., Haug, G.H., Haughen, K.A., Röhl, U., 2000, Rapid changes in the hydrologic cycle of the tropical Atlantic during the last glacial: Science, 290, 1947-1951.

Reimer, P.J., Baillie, M.G.L., Bard, E., Bayliss, A., Beck, J.W., Bertrand, C.J.H., Blackwell, P.G., Buck, C.E., Burr, G.S., Cutler, K.B., Damon, P.E., Edwards, R.L., Fairbanks, R.G., Friedrich, M.,Guilderson, T.P., Hogg, A.G., Hughen, K.A., Kromer, B., McCormac, F.G., Manning, S.W., Ramsey, C.B., Reimer, R.W., Remmele, S., Southon, J.R., Stuiver, M., Talamo, S., Taylor, F.W., van der Plicht, J., Weyhenmeyer, C.E., 2004, IntCal04 Terrestrial radiocarbon age calibration 26 - 0 ka BP: Radiocarbon, 46, 1029-1058

Rothwell, R.G., Rack, F.R., 2006, New Techniques in sediment core analysis: an introduction, in Rotwell, R.G. (ed.), New Techniques in Sediment Core Analysis (Special Publication No. 267): Londres, The Geological Society, 1-29.

Rothwell, R.G., Hoogakker, B., Thomson, J., Croudace, I.W., Frenz, M., 2006, Turbidite emplacement on the southern Balearic Abyssal Plain (western Mediterranean Sea) during Marine Isotope Stages 1-3: an application of ITRAX XRF scanning of sediment cores to lithostratigraphic analysis, in Rothwell, R.G. (ed.), New Techniques in Sediment Core Analysis (Special Publication No. 267): Londres, The Geological Society, 79-98.

Roy, P.D., Caballero, M., Lozano, R., Smykatz-Kloss, E., 2008, Geochemistry of late quaternary sediments from Tecocomulco lake, central Mexico: Implication to chemical weathering and provenance: Chemie der Erde - Geochemistry, 68, 383-393.

Serrano, H.D.E., 2004, Procesos termodinámicos en el lago volcánico de Santa María del Oro, Nayarit: México, D. F., Universidad Nacional Autónoma de México, Tesis doctoral, 94 p.

Stuiver, M., Reimer, P., 1993, Extended ${ }^{14} \mathrm{C}$ data base and revised Calib 3.0 14C calibration program: Radiocarbon, 35, 215-230.
Stuiver, M., Reimer, P.J., Reimer, R., 2005, Calib 5.0 program and documentation (programa informático, en línea): Belfast, Reino Unido, Queen's University Belfast, disponible en $<$ http://calib.qub. ac.uk/calib>, consultado 10 de noviembre de 2010 .

Tereshchenko, I.E., Filonov, A.E., Monzón, C.O., Figueroa, A., 2003, Weather signature of "E1 Niño" in western Mexico: Geofísica Internacional, 42, 389-396.

Therrell, M.D., Stahle, W.D., Acuña-Soto, R., 2004, Aztec drought and the "curse of one rabbit": Bulletin of the American Meteorological Society, 85, 1263-1272.

Therrell, M.D., Stahle, W.D., Villanueva-Díaz, J., Cornejo-Oviedo, Cleveland, M.K., 2006, Tree ring reconstructed maize yield in Central Mexico: 1474-2001: Climatic Change, 74, 493-504.

Valero-Garcés, B.L., Laird, K.R., Fritz, S.C., Kelts, K., Ito, E., Grimm, E.C., 1997, Holocene climate in the northern Great Plains inferred from sediment stratigraphy, stable isotopes, carbonate geochemistry, diatoms and pollen at Moon Lake, North Dakota: Quaternary Research, 48, 359-369.

Vázquez-Castro, G., 2004, Caracterización de los sedimentos del Holoceno tardío del lago Santa Maria del Oro, Nayarit, utilizando métodos de magnetismo ambiental: México, D.F., Universidad Nacional Autónoma de México, Instituto de Geofísica, Tesis de maestría, $153 \mathrm{p}$.

Vázquez-Castro, G., Ortega-Guerrero, B., Rodríguez, A., Caballero, M., Lozano-García, S., 2008, Mineralogía magnética como indicador de sequía en los sedimentos lacustres de los últimos $c a .2600$ años de Santa María del Oro, occidente de México: Revista Mexicana de Ciencias Geológicas, 25, 21-38.

Verma, S.P., Lozano-Santa Cruz, R., Girón-García, P., Velasco, F., 1996, Calibración preliminar de Fluorescencia de rayos X para análisis cuantitativo de elementos traza en rocas ígneas: Actas INAGEQ, $2,237-242$.

Villanueva-Díaz, J., Luckman, B.H., Stahle, D.W., Therrell, M.D., Cleaveland, M.K., Cerano Paredes, J., Gutiérrez-García, G., EstradaAvalos, J., Jasso-Ibarra, R., 2005, Hydroclimatic variability of the upper Nazas basin: Water mangement implications for the irrigated area of the Comarca Lagunera, México: Dendrochronologia, 22, 215-223.

Villanueva-Díaz, J., Stahle, D.W., Luckman, B.H., Cerano Paredes, J., Therrell, M.D., Cleaveland, M.K., Cornejo-Oviedo, E., 2007, Winter-spring precipitation reconstructions from tree rings for northeast Mexico: Climatic Change, 83, 117-131.

Villanueva-Díaz, J., Fulé, P.Z. Cerano Paredes, J., Estrada Ávalos, J., Sánchez Cohen, I., 2009, Reconstrucción de la precipitación estacional para el barlovento de la Sierra Madre Occidental con anillos de crecimiento de Pseudotsuga menziesii (Mirb.) Franco: Revista Ciencia Forestal en México, 34, 39-71.

Wang, C., Fiedler, P., 2006, ENSO variability and the eastern tropical Pacific: A review: Progress in Oceanography, 69, 239-266.

Manuscrito recibido: Octubre 15, 2010.

Manuscrito corregido recibido: Diciembre 1, 2010.

Manuscrito aceptado: Diciembre 8, 2010. 